\title{
Une esthétique de l'esquive : stratégies de résistance et de retrait dans l'espace sylvestre de Sylvain Tesson
}

Halia KOO, Memorial University of Newfoundland

\section{Introduction}

Dans Petit traité sur l'immensité du monde (2005), une réflexion empirique sans prétention théorique ou esprit de système sur l'avenir du voyage au vingt-et-unième siècle, Sylvain Tesson évoque une anecdote personnelle où il s'était farouchement - et en vain mobilisé contre l'abattage, sous prétexte de sécurité automobile, de platanes centenaires sur les routes françaises. Il en profite pour citer une phrase attribuée à Chateaubriand et qui fait partie des lieux communs de la doxa écologiste : «Les forêts précèdent les peuples et les déserts leur succèdent » $(147)^{1}$. Tesson, dont l'œuvre est marquée par les préoccupations écologiques et un discours anti-anthropocentrique, lance ensuite une boutade sur la théorie darwinienne selon laquelle l'Homme descend du singe : «Tous nos malheurs sont nés du fait d'avoir quitté nos arbres. Il est salutaire d'y retourner au moins de temps en temps. Pour retrouver nos racines, il faut remonter dans les branches » (Traité, 147). Pour montrer combien il fait peu de cas de la prétendue supériorité de l'être humain, il énonce cette formule mordante sur l'évolution de l'espèce humaine, qu'il considère comme une déchéance : «La Chute, ce fut lorsque l'homme descendit du singe » (Aphorismes, 110), puisque ce qui abaisse l'humain, c'est précisément son humanité. En même temps qu'un retour aux origines, la proximité des bosquets et le contact des feuillages favorisent un séjour dans un univers féerique et secret : pour Tesson, le fait de se perdre dans les buissons, de grimper aux arbres et de côtoyer les bêtes sauvages est un exercice vital qui permet de réenchanter le monde qui l'entoure, de «partir rencontrer les dieux dans sa forêt intérieure, de lâcher les chevaux de son imagination » afin de procéder à une «double lecture du monde » (Traité, 100).

Dans la conception dualiste de Tesson, le monde concret s'oppose en effet à une forêt secrète qui existe dans l'imagination de l'auteur comme une entité idyllique et hermétique, fragile et menacée. Avec la prise de conscience, à l'ère de l'Anthropocène, de la nécessité d'une réflexion et d'un questionnement à propos de l'impact de l'humanité sur 
l'environnement, l'œuvre de Tesson, qui consiste en grande partie de récits de voyage, se renforce d'une dimension écologique. L'auteur rejette en effet la conception humaniste et anthropocentrique de l'univers pour tenter de redéfinir un état de coexistence avec le monde qui puisse échapper aux limites imposées par la modernité. Il en résulte un certain pessimisme provoqué par le sentiment du dérèglement des rapports de l'humain avec son milieu naturel, ainsi qu'une mélancolie existentielle teintée de fatalisme qui tente de se ménager une échappée - ou une échappatoire - dans l'univers de la forêt. Il s'agit d'une attitude de repli qui oscille entre résistance et reddition, alors même que l'espace sylvestre finit par s'imposer comme un lieu de régénération physique et de reconquête intérieure.

\section{Le dernier refuge du rebelle solitaire}

Dans Forêts (1992), Robert Pogue Harrison note que dans l'imaginaire occidental, la forêt forme la lisière des espaces urbains et marque la limite du monde civilisé (ix-x) : l'espace sylvestre a toujours été considéré comme un territoire en marge de la société et de

ses lois. À la fois sphère sacrée et profane, la forêt a été représentée dans la mythologie et dans la littérature comme une zone mystérieuse où règnent l'anarchie et la confusion, le surnaturel et le merveilleux. Les phénomènes de déforestation et d'industrialisation renforcent cette distinction et consolident l'image de la forêt comme l'envers de la civilisation, un remède aux effets de l'urbanisation effrénée de notre époque, le dernier refuge du proscrit, du rêveur et du sauvage, ainsi qu'une destination de choix pour un écrivain-voyageur tel que Tesson. L'anthropologue Philippe Descola, dont le travail porte sur la critique du dualisme nature/culture, rappelle qu'il convient de faire une distinction « entre ce qui relève de la sylva, la forêt, en tant qu'espace fantasmatique du sauvage, et de la foresta, la forêt lorsqu'elle est cadastrée, aménagée, mise en valeur par la civilisation humaine » (6). En l'occurrence, la forêt recherchée par Tesson est bien la sylva idéalisée, sublimisée et poétisée, le lieu de la non-civilisation, le refuge des hors-la-loi, et l'espace porteur de tous les espoirs du randonneur nostalgique qui fuit les fléaux de la modernité.

Pour Tesson, auteur au prénom prédestiné, le retour à la forêt sauvage signifie avant tout se révolter contre l'asservissement de l'être humain par la technologie, l'illusion virtuelle, la réalité augmentée et le haut débit: «Bientôt, nous nous réfugierions dans les bois. Nous serions liés aux bêtes, aux forêts, à l'amitié, à nos morts, à nos livres. Nous 
serions déliés des machines. [...] Et nous serions vivants parce que déconnectés »(Oscillation, 119-120). Le mouvement de repli dans la forêt est motivé par le rejet de la société hyperindustrialisée et hyperconnectée, avec sa frénésie productiviste, son vacarme assourdissant et ses conflits destructeurs : « Recourir aux forêts, c'est tourner le dos à la laideur moderne », affirme Tesson, précisant qu'il s'agit du moyen « de fuir le monde le plus esthétiquement possible » (Traité, 152). La retraite vers la forêt représente également un retour allégorique à l'état originel et à l'univers fabuleux des mythes : «Se replier dans la forêt [...] est une réponse satisfaisante à la laideur du réel en même temps qu'un retour symbolique sous les frondaisons du monde onirique » (149). La forêt est présentée ainsi comme un espace tourné simultanément vers le passé et l'avenir, un territoire à la fois féerique et concret, qui renvoie aux angoisses immémoriales de l'homme mais lui prodigue en même temps une force énergisante : «Les bois : dernier endroit du monde où remontent à la surface de nos âmes perdues les vieilles terreurs et les nouveaux élans » (150). L'idée du merveilleux sylvestre est renforcée dans la description d'une promenade dans les bois, qu'il affirme être peuplés d'habitants du monde surnaturel comme les elfes et les lunanthropes (vampires et loups-garous), ainsi que les présences arboricoles comme les fantômes de pendus et les spectres de Waldgänger, «ces réprouvés qui avaient recours aux forêts quand la société des horizons ouverts ne voulait plus d'eux » (145) et qui choisissaient de démissionner du monde et de se retirer dans les bois, lieu d'asile et d'autonomie.

L'idée d'un « recours à la forêt » et la figure idéalisée du rebelle sont une référence directe à l'ouvrage Der Waldgänger d'Ernst Jünger (publié en 1951 et traduit sous le titre Le traité du rebelle, ou le recours aux forêts). Jünger - à qui Tesson voue une vénération particulière $^{2}$ - prône par ailleurs un individualisme anarchisant dressé contre la civilisation du machinisme et la suprématie du béton, et développe l'archétype du rebelle insoumis et hostile au despotisme de l'État-Léviathan ${ }^{3}$. La pensée jüngerienne s'inscrit à l'origine dans le sombre contexte des années suivant la Deuxième Guerre mondiale où l'humain, face à l'écroulement de ses convictions et de ses valeurs, doute de la validité des institutions, remet en cause la légitimité de l'État, et souhaite se forger une vérité personnelle pouvant le mener vers une forêt allégorique, zone de renaissance qui échappe à la destruction, un « lieu de la liberté [qui] est bien différent de la simple opposition ; ce n'est pas non plus l'un de ceux 
que l'on atteint par la fuite. Nous l'avons appelé la forêt » (Rebelle, 54), le lieu de refuge que l'humain libre peut rallier à l'heure des persécutions en vertu d'une décision délibérée et solitaire, plutôt que de se soumettre à l'embrigadement et à l'endoctrinement par la société :

C'est au fond de son cœur que l'homme doit chercher le code qui légitime ses actes et qui lui permet de «résister » aux forces inhumaines qui pèsent sur lui. Cet espace intemporel dans lequel est inscrit ce code, cette norme, il l'appelle la Forêt. C'est le «paysage » dans lequel se meut la Figure du Rebelle. (Beltran-Vidal 231)

Le mot Waldgänger, traduit par « rebelle » faute d'un équivalent français exact, trouve ses racines dans une ancienne coutume médiévale scandinave selon laquelle le proscrit ou horsla-loi avait « recours aux forêts » et s'y réfugiait loin de la communauté. Le Waldgänger de Jünger est un rebelle qui s'exclut de la société par libre choix et par protestation, une figure intemporelle qui peut être actualisée à tout moment de l'histoire et peut s'appliquer tant à Robin des Bois qu'aux chouans, camisards, résistants et francs-tireurs qui gagnent le maquis pour s'insurger contre le despotisme. On retrouve chez Tesson l'expression d'une résistance analogue au pouvoir absolu de l'État, qui exige de ses citoyens subordination, allégeance et conformisme. Tesson rappelle qu'afin d'asseoir son autorité et son contrôle sur le territoire, l'État pratique le déboisement, le défrichage, le déblaiement et la destruction de toute forme de végétation spontanée en vue de l'asservissement de la terre, des corps et des esprits : «Pour administrer un pays, la règle est de le défricher. Dans un royaume en ordre, la forêt est le dernier bastion de liberté à tomber » (Forêts, 168).

Dans l'exhortation que Tesson lance aux anticonformistes - « Refuzniks de tous les pays, gagnez les bois !»(169) - , on constate pourtant que ce qui est au départ une métaphore jüngerienne pour la résistance morale devient une recommandation à interpréter au pied de la lettre, une mise en application qui cadre parfaitement avec les thèmes écologiques traités par l'auteur. Dans Petit traité sur l'immensité du monde, il se promet de suivre « la piste des coureurs de bois postmodernes » (155) et de se retirer en ermite dans une forêt «nourricière et vide d'hommes » (150) : ce rêve, il le réalise en passant six mois dans une cabane isolée au bord du lac Baïkal, une expérience de solitude qui lui inspire Dans les forêts de Sibérie (2011). Toutefois, comme le montre Pierre Schoentjes, ce projet de détachement absolu s'avère une entreprise provisoire et précaire, « une posture que 
l'écrivain a bien du mal à maintenir dans la durée » (166). Car cette forme d'évasion est compromise par l'inévitable incursion de la modernité dans la tentative d'isolation et d'autarcie, et la sérénité recherchée par l'écrivain est régulièrement perturbée par la nécessité d'exprimer son inquiétude sur l'état de la planète, ainsi que son mépris de la société de consommation. Hannes De Vriese relève également qu'il existe dans le récit de Tesson des discordances et des contradictions qui remettent en cause la validité de son projet. Sa retraite dans les bois est envisagée d'abord comme un défi lancé à soi-même, mais elle finit par être compromise par un manque de sérieux et de sincérité (245). Du reste, les motivations de l'aspirant ermite sont passablement superficielles, et le récit se clôt sur un constat d'impuissance et une note de mélancolie. En fin de compte, la démarche de Tesson est problématique car elle est marquée par une «tension » entre écocritique et écopoétique (Schoentjes 166; De Vriese 247), c'est-à-dire un balancement entre deux modes d'analyse des représentations de la nature, l'écocritique se servant du point de vue de l'écologie politique pour exhorter à l'engagement actif, tandis que l'écopoétique met davantage l'accent sur les dimensions formelles des représentations littéraires et artistiques de l'environnement, ainsi que sur la contemplation esthétique de la beauté du monde naturel (De Vriese 235-236).

Si l'on considère uniquement Dans les forêts de Sibérie comme le récit d'une tentative d'engagement écologique, il est exact que cette aventure semble se solder par un échec (De Vriese 246). Néanmoins, en examinant l'œuvre de Tesson dans son ensemble, on remarque que sa désillusion et son pessimisme sont plus que la conséquence involontaire d'une expérience ratée : c'est un choix esthétique qui s'aligne sur le principe de la fuite consciente et la sauvegarde d'une liberté individuelle face au totalitarisme de la modernité ${ }^{4}$. Au demeurant, rien ne contraint le rebelle à fuir la société et à s'exiler dans la forêt, sa résistance relevant d'une mentalité et non d'un état physique conditionné par sa situation dans l'espace. La notion de « recours aux forêts » est ouverte à une éventuelle interprétation littérale et peut devenir sujet à expérimentation, sans que l'échec d'une telle expérience remette nécessairement en cause la position de Tesson en tant que Waldgänger. 


\section{Le reboisement symbolique du paysage urbain}

Confronté à la réalité d'une forêt qui se fait de plus en plus rare, Tesson s'efforce cependant de se créer une forêt de substitution en installant des bivouacs clandestins en plein centre-ville et à reconstituer, dans l'apparente stérilité du désert urbain, une nature imaginaire. Puisque Jünger affirme que pour le Waldgänger, « tout est forêt ; elle peut aussi se trouver dans le faubourg d'une grande ville » (Rebelle, 88-89) et que «même au sein d'une grande cité il existe des "forêts" de nature morale où l'on peut trouver asile » (Hervier 366), le rebelle écologique œuvre farouchement pour la concrétisation de ladite forêt symbolique, une mission clandestine qui prend parfois des allures d'opérations de guérilla écologistes. Dans sa tentative de réimaginer la forêt au milieu de la ville, Tesson plante sa tente sur les fortifications de Carcassonne, suspend son hamac aux poutrelles des ponts de Paris, installe son campement dans les arbres d'un square à Chartres, dort sur des bancs de parc de Bavière, ronfle sur les remparts de Saint-Malo, et grelotte sur le pavé à Paris, à Athènes et à Moscou. En digne adepte de la pensée jüngerienne, Tesson pratique cette forme de virée buissonnière dans un geste subversif et provocant, en réaction à l'immobilité, au statu quo et au conventionnalisme sédentaire imposés par l'ordre établi, puisque «le bivouac dérange l'État » (Traité, 133), incluant sa démarche dans un acte d'insubordination et de résistance.

Dans un même élan, Tesson s'adonne à la stégophilie (néologisme pour l'alpinisme urbain) : celui que ses camarades grimpeurs surnomment « le prince des chats » explore la ville en escaladant toitures, monuments et cathédrales, qu'il assimile dans ses textes à des crêtes montagneuses et à des terrains boisés. Selon lui, on peut déplorer inutilement la pénurie de nature en milieu urbain, ou « décider que les flèches gothiques qui piquettent l'Île-de-France deviendront ses propres Alpes » (Traité, 107). Mieux encore : pour lui qui se considérait un «alpiniste des villes», «les cathédrales étaient des Himalayas » (Oscillation, 45). Il explique le besoin de s'adonner à la randonnée verticale par une envie d'évasion en pleine nature : «Nous grimpions chaque fois que nous prenait l'envie de nous évader de la ville. La stégophilie [...] était notre salut, les clochers nos terres d'élection, les flèches nos rampes de lancement » (Traité, 106). Cette activité—forcément nocturne car clandestine, au même titre que le bivouac-lui permet de trouver encore, sur une planète explorée et défrichée jusque dans ses moindres recoins, des territoires inconnus 
à découvrir. Les cathédrales deviennent ainsi « des jungles obscures, [...] des îles de pierre à explorer » (105).

Un thème récurrent dans l'œuvre de Tesson est la notion selon laquelle le caractère envahissant et omniprésent de la modernité dans la société contemporaine ne saurait être parfait, et que cet écran en apparence impeccable tendu entre l'homme et son milieu naturel comporte des défauts et des fêlures par lesquels on peut s'engager pour atteindre une forêt imaginaire, et qu'il revient au voyageur de découvrir. À l'image de Jünger qui évoque la nécessité pour le rebelle de trouver «l'entrée des jardins intemporels » (Rebelle, 145), le marcheur doit «s'infiltrer dans ce qui restera d'interstices de liberté, d'autrements » face à la pénurie de ce que Christian Verrier nomme «lieux-refuges » ou «lieuxautrements »(157) qui permettent l'exil volontaire. On observe ainsi, chez Tesson, l'utilisation répétée des mots «fissures », «brèches », « replis », « interstices » et « failles » qui permettent de repérer l'entrée cachée de la forêt enchantée. Dans Petit traité sur l'immensité du monde, il déclarait déjà à propos du bivouac en milieu urbain qu'il n'est « point besoin de campagne bocagère pour bivouaquer. La ville offre des replis savants à celui qui sait les débusquer »(132). L'ascension des cathédrales participe de même à ce projet de reconstitution d'une réalité archaïque et lointaine, devenue un mythe inaccessible.

Seul ou en groupe, Tesson s'est offert plusieurs de ces excursions en haute altitude et ces escapades dans le vide, repérant les passages praticables, localisant les points faibles et les lignes de force de l'église. Avec ses amis, il forme une bande de joyeux lurons qui a allègrement «dormi sous les toitures, dîné dans les nefs, trinqué dans les clochetons »(Traité, 111). Mais au-delà de sa dimension funambulesque, cette gymnastique périlleuse aide à reconstituer une forêt imaginaire, un univers secret et marginal qui est perçu comme un reflet du relief urbain, avec un système d'équivalence des éléments topographiques :

Nous avons ainsi élaboré une représentation géographique parallèle des villes de France, à un niveau supérieur, superposable aux plans officiels ; une géographie du vide avec son océan de toits - tuiles, ardoises et zinc: variété de la nappe ! - entaillé par des failles (les rues), ses archipels formés par les coupoles, ses pics en forme de clochers. (124)

Grâce à la stégophilie, Tesson affirme avoir trouvé « des chemins de traverse dans la ville - des failles dans la citadelle - où se glisser savamment. Ces chemins mènent au cœur de 
jardins féeriques » (Traité, 104). Dans Marche avant (2011), Alexandre Poussin, camarade de voyage et compagnon de cordée de Tesson, parle également de la «forêt » (387) de Notre-Dame de Paris et ne manque pas d'en évoquer l'allure féérique : en décrivant l'ascension de la cathédrale, Poussin déclare que « [n] ous sommes en pleine féerie » (389), avant de comparer l'alpiniste urbain (qui a réussi à déjouer tous les pièges et obstacles que présente un édifice gothique) à un héros de conte de fées, un «prince charmant bataillant contre la forêt de ronces maléfiques » (387). En notant que «jardin féerique » est le nom que l'alpiniste Gaston Rébuffat donnait au massif du Mont-Blanc avec ses crêtes et ses flèches granitiques, Tesson choisit d'attribuer ce nom à «d'autres massifs de pierre, à d'autres forêts de flèches: aux cathédrales gothiques des grandes capitales européennes » (Traité, 104).

\section{La forêt cathédrale, et la cathédrale forêt}

Sensible à l'esthétique et au symbolisme des cathédrales médiévales, Tesson les qualifie de «jardins de pierre » (Traité, 105) et de « forêt[s] » (115). De fait, la «forêt » est le nom que l'on donne communément à la charpente de la nef de la cathédrale Notre-Dame de Paris, car la totalité de cette ossature impressionnante représente l'équivalent d'une forêt de chênes de vingt-et-un hectares, chaque poutre provenant d'un arbre différent. Tesson précise : «On devrait dire la jungle car c'est un enchevêtrement de poutres ajustées les unes aux autres sans rivets, ni chevilles : un mikado de châtaigniers » $(115)^{5}$. De la même façon, Harrison compare la cathédrale gothique aux anciennes forêts de l'époque païenne, et établit une analogie entre nature et architecture qui rejoint la perception de Tesson. Harrison évoque la nature symbolique de la forêt, considérée par les barbares germaniques comme le berceau de leur race. Il fait allusion à un traité de l'historien Tacite, qui décrit la forêt hercynienne comme un lieu vénéré par les indigènes en tant que demeure du dieu suprême, et un lieu propice aux apparitions divines. Harrison fait remarquer à ce propos que le caractère sacré de la forêt confère une signification toute nouvelle à l'expression «the cathedral forest » (la futaie cathédrale), une forêt d'arbres très élevés :

The Gothic cathedral visibly reproduces the ancient scenes of worship in its lofty interior, which rises vertically toward the sky and then curves into a vault from all sides, like so many tree crowns converging into a canopy overhead. [...] In other words, the phrase "cathedral forest" entails more 
than just a casual analogy ; or better, the analogy has its basis in an ancient correspondence between forests and the dwelling place of a god. (178)

Tesson lui-même n'est pas croyant au sens traditionnel du terme : ses textes affichent généralement un scepticisme ironique à l'égard de la religion organisée, et il écrit que «[s]on église n'a qu'une voûte : celle des arbres »(Aphorismes, 137). La forme de spiritualité qu'il privilégie, est celle de la possibilité d'une expérience mystique dans la nature sauvage et, considérant les églises gothiques comme un substitut à la forêt sacrée, il distingue dans l'aspect, la structure et la disposition des cathédrales des analogies non seulement sylvestres, mais aussi géologiques, entomologiques et astrales qui renvoient à l'ordre de l'univers. Il relève par exemple que selon la tradition, la science architecturale des églises médiévales dans la région parisienne aurait un fondement cosmique, en communion avec les lois de la nature : «elle use des mêmes lois que celles qui régissent la course des planètes » (Traité, 108). La disposition des églises et basiliques en cercle autour de Notre-Dame de Paris «dessine sur la carte, en miroir, la constellation de la Vierge » (108). En outre, Tesson identifie dans les toits en flèche qui tendent vers le ciel des propriétés dynamiques productrices d'énergie, les comparant à une source minérale d'où jaillit la vie : «Une flèche est un geyser de sève minérale. Les moellons de l'édifice entier, parcourus par les flux montants, sonnent comme le cristal si on les frappe de l'ongle » (112).

La cathédrale elle-même est envisagée comme un écosystème : Tesson fait observer que comme toute bonne forêt, les cathédrales sont peuplées d'animaux farouches qui forment «un double bestiaire »(118). D’un côté, un bestiaire enchanté composé de monstres, gargouilles et griffons de pierre suspendus dans le vide, «les vigies des cités » (118) qui président aux destinées urbaines et ne s'animent qu'à la lueur des lampes. D'autre part, un bestiaire bien réel, des créatures qui n'ont rien de chimérique et qui servent de guides aux grimpeurs : chouettes, faucons, chauves-souris, loirs, furets, araignées et pigeons. Naturellement, «[i]l n'y a nulle part de bestiaire sans flore. Pas moins dans les cathédrales qu'ailleurs » (119), comme en témoigne la présence de buissons et de petits arbres qui sont parvenus à s'infiltrer dans les fissures de la pierre, exprimant d'une façon métaphorique la revanche de la plante sur le béton. Bientôt, Tesson se surprend à entretenir l'utopie de voir un jour la ville envahie par la forêt qui reprend ses droits sur le territoire : 
«Une promenade attentive dans la ville permet de ne pas désespérer de l'avenir des plantes »(120), assure-t-il, avant de se lancer dans une envolée lyrique sur la lente et imperceptible conquête de l'espace urbain par le règne végétal :

On se fout des herbes folles. Jamais un regard accordé aux frémissements du végétal. Pourtant, on devrait prendre garde. Ces présences à peine visibles attendent peut-être leur heure. Il est possible qu'elles occupent le terrain avant le grand assaut et qu'un jour elles déchaînent leurs forces pour reconquérir la ville qui les a spoliées du ciel. Le raz-de-marée de sève engloutira alors les habitations. Et le prochain règne sera végétal. (120-121)

Dans cette vision non plus apocalyptique mais édénique d'une société industrielle reconquise par la végétation, Tesson se surprend à nourrir l'espoir chimérique d'une forêt qui empiète sur la ville.

\section{Les forêts du recours, les forêts du secours}

Dans ce jardin paradisiaque illusoire survient pourtant un jour la chute de l'homme : en août 2014 à Chamonix, après avoir fêté la remise du manuscrit de son nouveau livre, Tesson tente d'escalader la façade d'une maison et fait une chute de dix mètres. Bilan de cet accident: sévère traumatisme crânien, enfoncement de la cage thoracique, colonne vertébrale endommagée, une vingtaine de fractures, paralysie partielle du visage, perte de l'ouïe à l'oreille droite, et des crises d'épilepsie intermittentes qui ne le quittent plus. Le corps brisé, Tesson n'est même pas certain de pouvoir marcher à nouveau. Mais après quatre mois d'hospitalisation, il se lance le défi de traverser la France à pied et d'aller à la rencontre d'une campagne méconnue, en empruntant des chemins cachés et des pistes à ornières qui traversent un territoire épargné par l'urbanisme et la technologie : un retour à la forêt véritable et un retour inéluctable aux sources. Il raconte cette pérégrination dans son récit Sur les chemins noirs (2016). «C'était un voyage né d'une chute » (140), reconnaîtil, un périple entrepris pour exorciser le traumatisme de son accident survenu dans la forêt urbaine et chercher l'apaisement dans la nature, ainsi que pour faire le deuil de sa mère, disparue la même année. Bien que physiquement diminué, il se donne comme objectif de marcher lentement du Mercantour au Cotentin, dans la campagne identifiée dans un rapport officiel de 2011 comme des zones hyper-rurales que l'enclavement, la faible densité de population et la faiblesse des infrastructures rendent réfractaires au développement économique. Dans ces zones soi-disant sous-développées, Tesson évite les agglomérations, 
les routes balisées et les sentiers de grande randonnée. Il suit les pistes pastorales oubliées, des voies antiques à peine entretenues, souvent laissées à la circulation des bêtes : ce sont les fameux « chemins noirs ».

Cette nouvelle retraite sous les bocages est plus qu'une expérimentation écologique ou une « parenthèse écopoétique » (De Vriese 235$)^{6}$ comme dans les forêts de Sibérie : il y entre un peu moins d'affectation et plus de simplicité, puisqu'il est question d'une épreuve physique intensément personnelle vécue dans la souffrance, en même temps qu'une épreuve psychique à surmonter quand guettent les regrets et le découragement. Le récit est ponctué d'épisodes dépressifs où Tesson développe une réflexion douloureuse sur sa nouvelle condition d'homme handicapé et défiguré qui traîne sur les routes un corps endolori et abruti par les médicaments. Les substances toxiques qu'il doit ingurgiter quotidiennement pour traiter ses douleurs et blessures sont telles qu'elles lui inspirent cette réflexion humoristicoécologique teintée d'autodérision: «Tâchons de ne pas tomber à l'eau, pensais-je en passant les ponts sur les ruisseaux, cela évitera à la région une pollution chimique »(Chemins, 103). Tout au long du récit, Tesson livre une méditation mélancolique dénuée de pathos sur sa propre vulnérabilité, mais aussi sur la fragilité d'une campagne bousculée par la mondialisation, mettant le lectorat en garde contre le danger de l'industrialisation forcenée qui menace l'environnement.

Tesson se retrouve malgré lui confronté aux réalités d'une campagne moribonde qui souffre des retombées négatives de la modernité : avec la mécanisation de l'agriculture et l'intensification de la production, la politique agressive des Trente Glorieuses a provoqué l'augmentation de la rentabilité et apporté une prospérité temporaire, mais la mondialisation a vite fait de rendre anachronique cette ruralité artificielle. Tesson avoue son incapacité à analyser ou critiquer la situation : «Je n'avais pas mené l'étude nécessaire à comprendre la mécanique de ces phénomènes ni ne disposais de la puissance intellectuelle pour les analyser » (102). Mais ses rencontres avec les rares fermiers et agriculteurs qui subsistent encore dans ce pays «presque effacé » (101) de la carte lui révèlent les conséquences d'une modernisation trop rapide qui a hâté la crise et a radicalement bouleversé l'équilibre entre l'être humain et son environnement: ce phénomène a engendré «un nouveau paysage, redistribué les cartes du sol, réorchestré la conversation de l'homme et de la terre » (46). Dans ces zones grises vidées par l'exode rural, on ne trouve que villages désertés, champs 
abandonnés, commerces fermés, maisons à vendre et habitants désabusés qui subissent, en première ligne, les tourments des mutations amenées par le marché global.

Contrairement à la nature sauvage des forêts de Sibérie, le paysage campagnard que décrit Tesson est une nature dénaturée par la modernité, et y trouver les chemins noirs est une tâche ardue : il faut, comme pour le bivouac et la stégophilie, repérer les brèches dans le mur: «Trouver les chemins noirs dans une campagne plus modernisée et jacobine compliquait le jeu. Il faudrait mieux scruter les lieux pour y repérer les caches » (109). « La France rurale se maintenait dans les replis » (141), et les chemins noirs sont des «contreallées » (35) et des « [p]assages secrets » (34) qui gardent le souvenir de la vieille France piétonne. Le voyage devient un travail de prospection qui consiste à vérifier «l'existence de contre-allées, inconnues, au cœur de la citadelle, de portes dérobées, d'escaliers de service où disparaître » (33), à trouver « des vallons où s'engouffrer » et des « interstices » (142) par lesquels s'infiltrer, et à repérer le passage dérobé qui mène à la forêt profonde, en quête d'un pays « illégal et irréel » (76) que l'urbanisation généralisée de notre époque rend illicite. Les chemins noirs sont en outre une anomalie spatiotemporelle, un vestige antique et « $[\mathrm{u}]$ ne fissure dans le présent » (49) ayant la capacité de tracer « la cartographie du temps perdu » (71). Dans un monde où tout a déjà été découvert, Tesson part à la recherche de décors sauvages et de territoires méconnus : avant sa chute, Tesson le stégophile déclarait déjà que «[g]rimper sur le dos d'une cathédrale, c'est s'avancer dans une terra encore un peu incognita » (Traité, 114). Tesson le randonneur n'a pas abandonné l'espoir de «tracer une sinusoïde de l'incognito » (Chemins, 34) dans la campagne française. Si les voyages de Tesson se situent entre soif du merveilleux et quête de l'inexploré, ils constituent aussi un geste de résistance et un acte d'insubordination envers l'ordre établi, entrepris pour échapper aux conventions et trouver des espaces libres des contraintes de l'administration, des «zones franches épargnées par la politique du territoire »(27). Les chemins noirs sont des «cheminements mentaux que nous emprunterions pour nous soustraire à l'époque » (35) et élaborer « une cartographie mentale de l'esquive » (35), un tracé qui lui permet de fuir les clameurs et les trépidations de la modernité, d'échapper aux conventions et de devenir invisible au monde extérieur : «en somme, se détourner. Mieux encore ! disparaître » (35). 


\section{Du rebelle à l'anarque : une apologie de la nostalgie}

L'exercice de disparition pratiqué par Tesson pour se retrancher de la société industrialisée est une «disparition désirée, antidote contre la servitude volontaire »(Chemins, 91), qui pose cependant le problème de la responsabilité et de l'engagement de l'écrivain. En 2010, dans les forêts de Sibérie, Tesson déplorait déjà son manque de courage à affronter la réalité, craignant que ses voyages et ses retraites ne servent que d'écran au défaitisme et à la poltronnerie :

La nostalgie, la mélancolie, la rêverie donnent aux âmes romantiques l'illusion d'une échappée vertueuse. Elles passent pour d'esthétiques moyens de résistance à la laideur mais ne sont que le cache-sexe de la lâcheté. Que suis-je ? Un pleutre, affolé par le monde, reclus dans une cabane, au fond des bois. Un couard qui s'alcoolise en silence pour ne pas risquer d'assister au spectacle de son temps ni de croiser sa conscience faisant les cent pas sur la grève. (Forêts, 180)

En 2007, dans Éloge de l'énergie vagabonde, il reconnaissait également que « [1]a nostalgie est une paresse » (136). Lorsqu'il suit les chemins noirs en 2015, le pessimisme écocritique subsiste, mais l'inquiétude de n'être qu'un contemplateur résigné évitant de s'impliquer pour la bonne cause est atténuée par une apologie de la résistance intérieure et du renoncement face aux calamités de l'ère contemporaine. Les séquelles de son accident et son état de santé précaire y sont pour quelque chose dans la réévaluation de sa position : alors qu'il parcourt les monts du Cantal en compagnie de son ami Arnaud Humann, il est victime d'une crise d'épilepsie provoquée par les fractures de son crâne, ce qui nécessite une brève hospitalisation et lui fait redouter l'éventualité d'une nouvelle attaque. Pris d'une angoisse viscérale et d'une mélancolie dépressive que semble aggraver la tristesse du milieu ambiant, Tesson se montre impatient de fuir ce paysage corrompu et fantomatique : «il nous fallait vite retrouver un chemin bordé d'arbres, nous rejeter sous les futaies, rejoindre un cours d'eau, sortir des zones livides »(Chemins, 100).

Étais-je en train de virer nostalgique ? Humann en était persuadé.

— Tu es gâteux, me disait-il.

— Et après ? lui répondais-je.

Était-il indigne de penser qu'un village avec flonflons d'orchestre et meuglement de bestiaux contenait davantage de poésie qu'une rue ventée où clignotaient des enseignes de manucures? J'avais été jusqu'alors l'ennemi de la pensée passéiste. Craignant la ringardise, j'avais considéré la nostalgie comme une maladie honteuse, pire que la cirrhose du foie, laquelle passait encore pour le prix des nuits joyeuses. (100) 
Dans un milieu rural artificiel qui n'est plus qu'une caricature grossière du paysage urbain, la forêt permet de cultiver l'illusion d'un rêve encore intact, loin de la réalité affligeante du monde. Dans une attitude de défi, au risque de frôler le passéisme égocentrique ou le conservatisme réactionnaire, Tesson choisit de revendiquer la nostalgie en tant que posture moralement défendable et esthétiquement acceptable - un processus qu'il qualifie de « réforme » :

les derniers mois m'avaient changé et cette courte marche dans le décor du pays avait accéléré la réforme. Je n'aurais plus honte désormais de m'avouer nostalgique de ce que je n'avais pas connu. [...] Était-il malséant d'établir une hiérarchie entre les choses ? De préférer la France de Roupnel - fût-elle fantasmée - aux alignements des maisons mortes ? (Chemins, 101)

Au lieu d'être négativisé, le sentiment de mélancolie et de découragement est magnifié et reconverti en une esthétique de l'esquive et de la rêverie écopoétique, susceptible d'apporter une satisfaction individualiste et solitaire. Cette forme de fatalisme assumé se précise dans un épisode où Tesson et son camarade suivent une voie ferrée désaffectée depuis près de cinquante ans et se fraient un chemin à travers les fourrés, le long des rails encombrés de ronces et de broussailles : «Le moment était romanesque : un chemin se perdait et nous nous y sentions bien car il n'offrait aucun espoir. Seulement le jaillissement des songes »(116: je souligne). Dans cette expression d'un désespoir heureux teinté de sentimentalité romanesque se résume une tentative de justification, de légitimation et d'esthétisation de l'attitude de démission.

La difficulté de Tesson à prendre position (dans les forêts de Sibérie d'abord, sur les chemins noirs ensuite) s'explique avant tout par le statut transgressif et ambivalent de la figure du rebelle. Dans Éloge de la marche (2000), David Le Breton évoque la position ambiguë du marcheur moderne qui recherche la solitude et le recueillement dans la nature. Par l'originalité et l'excentricité de sa démarche, il se démarque des autres et se place dans un espace intermédiaire qui n'est pas aisément catégorisable : «Le marcheur est un homme de l'interstice, de l'entre-deux, son emprunt des chemins de traverse le situe dans l'ambivalence d'être à la fois dehors et dedans, ici et là » (90). Un marcheur rebelle tel que Tesson, exilé volontaire anticonformiste, appartient certes au monde de l'humain, mais il est tout autant autre et ailleurs par sa retraite sylvestre et son exploration des brèches et des 
fissures, évoluant «différemment, cultivant et préservant, malgré [lui] peut-être, une sorte de contestation, de protestation calme, ne serait-ce que par [son] décalage » (Verrier 157).

En outre, l'attitude ambivalente de Tesson reproduit un modèle idéologique évolutif que Jünger élabore pendant plusieurs décennies et qui repose sur les figures du Rebelle et de l'Anarque, principalement développées dans le traité philosophique Der Waldgänger et le roman utopique/dystopique Eumeswil (1977). En 1951, pendant la période de reconstruction de l'après-guerre, alors que l'Europe se relève de l'épisode traumatisant des totalitarismes meurtriers, Jünger écrit à propos de la liberté revendiquée par le Waldgänger qu' «il ne s'agit pas seulement de cette liberté qui proteste ou émigre, mais d'une liberté qui décide d'engager la lutte. [...] Le Rebelle ne peut se permettre l'indifférence » (Rebelle, 102) car sa résistance est absolue : «elle ne connaît pas de neutralité » (Rebelle, 103). Cependant, lorsque Eumeswil paraît en 1977, la vision du Waldgänger a évolué et s'est adaptée au contexte sociopolitique de l'époque qui voit la démocratisation de l'Allemagne de l'Ouest. L'intrigue d'Eumeswil se déroule dans une cité-État à régime autocratique mais relativement tolérant : le héros est un «anarque » qui, sans être anarchiste, est indifférent aux clivages politiques et se méfie de «[1]'illusion égalitaire des démagogues [qui] est encore plus dangereuse que la brutalité des traîneurs de sabre » (262), un comportement qui exprime autant le désenchantement que Jünger éprouve à l'endroit des démocraties libérales que son aversion pour les dictatures répressives. L'anarque est un observateur impassible mais vigilant qui maintient un équilibre subtil entre résistance silencieuse et mépris tranquille des institutions politiques, pour disparaître à la fin dans une forêt impénétrable dont il ne reviendra pas. Dans cet ouvrage, Jünger renonce à l'usage du terme Waldgänger et élabore la figure de l'anarque qui prend le relais du rebelle (Hervier 466) et représente l'idéal de l'individu souverain capable de conserver sa liberté de pensée et qui choisit de se retirer dans la forêt. Jünger explique ainsi la distinction entre ces deux états d'esprit :

il ne faut pas confondre rebelle (Waldgänger) et partisan; le partisan se bat en compagnie, le rebelle tout seul. Le rebelle ne doit pas non plus être confondu avec l'anarque, bien que l'un et l'autre soient parfois très semblables et difficiles à distinguer sur le plan existentiel. La distinction réside en ce que le rebelle a été banni de la société, tandis que l'anarque a banni la société hors de lui-même. Il est et reste son propre maître en toutes circonstances. S'il se décide à recourir aux forêts, c'est moins pour lui une question de droit et de conscience qu'un accident de parcours. Il change de camouflage. (Eumeswil, 155-156) 
L'anarque a comme particularité d'affecter un certain respect extérieur des lois en tant que conventions sociales, mais de se garder d'éprouver du respect à leur égard. Dans la mesure où ces régimes politiques n'ont rien de totalitaire, Jünger considère que l'anarque peut être « un virtuose de la restriction mentale » (Hervier 478) résolu à conserver son équanimité et ses propres exigences morales et à se dérober à une réalité affligeante, comme par exemple la querelle qui oppose économistes et écologistes, un thème qui montre à quel point Jünger était en avance sur son temps : dans l'univers post-apocalyptique d'Eumeswil, « des conflits éclatèrent entre l'environnement humain et l'environnement naturel » (Eumeswil, 400), c'est-à-dire entre ceux qui cherchent à répartir les fruits de la croissance et ceux qui tentent d'assurer la survie du milieu naturel en prônant la décroissance.

Dans la perspective écologique qu'adopte Tesson, sa «stratégie de la rétractation »(Chemins, 140) est un choix qui correspond à l'évolution de la pensée jüngerienne, et la tension entre écocritique et écopoétique décelée précédemment chez Tesson est en somme le reflet du continuel tiraillement idéologique entre l'attitude du rebelle et celle de l'anarque. Dans un texte où il analyse les similitudes et les différences entre l'anarque et le rebelle, Patrick Louis précise que

l'anarque [est] celui qui a su maîtriser sa propre anarchie intérieure [...]. L'Anarque ressemble au Rebelle. [...] Comme lui, il se veut serviteur de la liberté au nom de l'ordre vrai. Comme lui, il est passé à la clandestinité, aux forêts. Mais le Rebelle est un combattant, il agit contre la tyrannie qui l'avilit, prêt à frapper dès que l'occasion s'en présente. L'Anarque a renoncé au combat, il a choisi l'émigration intérieure. (59-60)

Puisque c'est une illusion de croire que l'on peut sauver le monde, la meilleure «solution à nos tristesses »(Traité, 152) réside dans l'émigration intérieure et la «cavale bocagère »(Chemins, 134). Tesson fait l'apologie de la rétraction et du repli, en reconnaissant l'impuissance de ses actes : «le recours aux forêts [...] semble être la seule chose qui reste à faire quand on a tout essayé » (Traité, 152). Évoquant les maux de la société moderne, Tesson revendique le droit au pessimisme et déclare se méfier des optimistes, et leur préférer « l'agitation, la fuite, l'esquive. Une lâcheté, l'escapisme ? Peutêtre. Mais je m'en moque. Fuyons, puisque demain sera pire qu'aujourd'hui » (Oscillation, 15). 
Il entre, dans la stratégie de retrait de Tesson, une part appréciable d'autodérision et d'autocritique : il est conscient que les actes et les pensées d'un ermite temporaire comme lui n'auront aucun poids dans le cours des choses, comme il le reconnaît lors de son séjour en Sibérie : «La cabane permet une posture, mais ne donne pas un statut. On joue à l'ermite, on ne peut se prétendre pionnier » (Forêts, 198). De même, il est probable que l'anarque n'aura aucune influence déterminante, aucune incidence notable sur les événements : «Qui sera sauvé par l'Anarque? La réponse s'impose : rien, ni personne, même pas lui-même [...]. L'Anarque est statique ; le Rebelle, dynamique. L'Anarque est un Rebelle désabusé, reflet du désabusement de Jünger vis-à-vis de toute cause politique » (Louis 60).

\section{Le retour à la forêt et à la terre : responsabilité et réciprocité}

L'apparente démission de l'anarque et l'absence d'engagement actif n'excluent pourtant pas la prise de conscience d'une responsabilité éthique vis-à-vis de la nature. Dans la vision du monde de Tesson, la désaffection de l'anarque pour la politique et son indifférence pour le militantisme ne le dispensent nullement de l'obligation de contribuer au bien commun en participant au fonctionnement et à l'équilibre de l'écosystème sylvestre, à plus forte raison si la forêt lui procure consolation et apaisement, et lui apporte de surcroît la guérison. Sur les chemins noirs, Tesson note que les nuits de bivouac passées au pied d'un arbre et les réveils en pleine nature ont la faculté d'opérer une redynamisation et une rééducation du corps et de l'esprit : «Ma stratégie du retrait distillait sa jouvence dans mes fibres »(Chemins, 41), car l'organisme humain est régénéré par l'air vivifiant, la terre nourricière et les arbres qui lui communiquent leur sève et leur énergie :

Une seule nuit sèche et tiède sous des arbres assez vieux avait opéré son miracle. Je me réveillai au pied d'un pin, gonflé d'une excitation nouvelle. L'arbre fait-il percoler un peu de sa force dans l'organisme de celui qui dort à son pied ? Après tout, on gagnait à rester dans le voisinage de certains êtres. Peut-être en allait-il de même avec les arbres ? (98-99)

Vécue comme un espace de régénérescence physique mais aussi de communion avec la nature, la forêt permet ainsi de renouer avec son milieu naturel et de retrouver « les racines qui relient notre chair à l'humus du monde » (Traité, 153). L'univers est représenté comme un organisme dont tous les membres existent en corrélation et se doivent d'être solidaires : «La forêt est un organisme total, composé de milliers d'individus. Chacun est appelé à 
naître, à vivre, à mourir, à se décomposer - à assurer aux générations suivantes un terreau de croissance supérieur à celui sur lequel il avait poussé. Chaque arbre reçoit et transmet » (Oscillation, 171-172), un devoir de réciprocité et de don de soi s'appliquant également à l'espèce humaine, qui est tenue de transformer ce qui n'est qu'un rapport de dépendance et d'exploitation en un lien d'interdépendance. De façon révélatrice, le chapitre «Les forêts du recours » de Petit traité sur l'immensité du monde se clôt sur une méditation sur le devoir de l'humain envers la nature en général, et celui du voyageur en particulier :

Je m'interroge [...] sur le prix que nous devrons payer à la planète en la quittant. C'est que j'ai horreur de me sentir débiteur. Puisque nous ne faisons qu'emprunter depuis le premier jour de notre existence, il serait juste de s'acquitter; pour alléger un peu sa dette. Le vagabond est plus redevable encore que les autres car non content de cueillir les fruits du monde, il a passé sa vie à se gorger de ses beautés. Et, quand vient la mort, il devrait se sentir étreint par l'angoisse de l'ardoise. (166-167)

Ce commentaire Tesson rejoint l'idée que les êtres humains ne peuvent que mener une existence qui est définie par un comportement de prédateur, ou « une existence qui ne peut être maintenue sans se nourrir de l'existence d'autres êtres vivants» (Lestel 67) ${ }^{7}$. Dominique Lestel, qui examine les rapports entre les créatures humaines et non-humaines, évoque la «dette infinie et personnelle» $(67)^{8}$, c'est-à-dire la dette immémoriale et incommensurable contractée auprès des animaux et de l'écosystème, ou «les obligations de réciprocité qui rattachent les hommes aux animaux et aussi aux plantes, aux champignons, aux intelligences incorporelles, etc. » $(67)^{9}$. La conscience d'avoir contracté une dette intrinsèque envers d'autres êtres vivants est un thème récurrent dans l'œuvre de Tesson : comme cette dette constitue le fondement même de la nature humaine et qu'il est impossible de s'en acquitter tout à fait, l'auteur décrit le sentiment de culpabilité et d'angoisse existentielle qui lui fait souhaiter pouvoir rembourser une part modeste de sa dette. Une possibilité serait d'élire sa dernière demeure sous un arbre afin de verser son écot et payer au moins une petite portion de sa «dette infinie », afin de boucler la boucle et participer au cycle de vie :

Ma dernière volonté sera d'être enterré sous un arbre que mon corps contribuera à nourrir. Ce sera ma manière de m'absoudre. J'aurai assez dévoré de viande pour donner la mienne, en juste retour, à des asticots. [...] L'arbre poussera auprès de ma dernière cabane. Mon corps alimentera la sève qui pulsera dans le tronc et peut-être qu'un oiseau posé sur une branche lancera un trille qui guidera un vagabond égaré vers ma cabane. (Traité,167) 
Cette solution, suggérée avec une ferveur toute écopoétique, va jusqu'à se soucier de la génération suivante d'ermites réfractaires. Mais elle se décline ailleurs sous la forme plus directe d'une description crûment scientifique du recyclage des matières corruptibles en vue de la fertilisation de la terre : «J'aimerais bien me faire enterrer sous un arbre. La chair, se décomposant, serait aspirée dans le système ligneux, distribuée dans les fibres jusqu'aux nervures des feuilles. Je rendrais ainsi à la nature ce que je lui ai raflé » $(\text { Oscillation, 173 })^{10}$. Par cette déclaration à la fois idéaliste et pragmatique, d'une ambivalence qui lui est typique, Tesson affiche l'ambition d'alimenter, dans un geste de gratitude et d'expiation, et par la biodégradation de son propre corps, une forêt décimée par l'humain.

L'image de la forêt, ultime refuge du rêveur solitaire et sanctuaire de la liberté individuelle, est habilement récupérée par Tesson à des fins de critique environnementale et sociale. Toutefois, il se défend de toute rébellion active ou de militantisme écologique, et il soutient qu'il ne cherche pas à changer le monde, mais simplement à l'éviter : « [le vagabond éternel] ne veut pas se battre, il s'échappe » (Traité, 54), explique-t-il pour justifier ses expéditions solitaires en milieu sauvage. Il n'attribue ainsi aucune signification politique à la vie en cabane, estimant que les idéologies n'ont pas leur place dans le refuge d'un ermite, et qu'il n'est pas de son ressort d'apporter une solution à l'impasse écologique : «En cabane, on vit à l'heure contre-révolutionnaire. [...] Vivre ici n'apporte rien à la communauté des hommes. L'expérience de l'ermitage ne verse pas son écot à la recherche collective sur les moyens de faire vivre les gens ensemble » (Forêts, 190). L'évasion dans la forêt s'inscrit clairement dans une stratégie de l'esquive, qui consiste à se tenir « à l'écart, dans un refus poli. [...] L'ermite ne s'oppose pas, il épouse un mode de vie. [...] Il est physiquement inoffensif et on le tolère comme s'il appartenait à un ordre intermédiaire, une caste médiane entre le barbare et le civilisé » (148).

\section{Conclusion}

Les textes de Tesson ne proposent pas de solutions tangibles aux problèmes sociaux et environnementaux de la planète, manifestant plutôt un manque d'activisme concret et même une tendance à l'épanchement lyrique. Sa position découle d'un choix qui admet que toute résistance est vaine et qui renonce à adopter une attitude combative et constructive, mais ce faisant, sa résistance intérieure et son détachement lucide contribuent à tempérer le 
ton de protestation indignée d'un rebelle activiste. Par son aveu d'impuissance, l'anarque solitaire fait de son calme désespoir un outil de persuasion qui prête une forme d'éloquence particulière à son plaidoyer pour une forêt en voie de disparition. Son obstination invincible et son fatalisme aux aguets donnent lieu à une perspective critique teintée de mélancolie qui s'abstient d'adopter une position de supériorité et évite le discours moralisateur, tout en proposant une réflexion engagée qui interpelle le lecteur et l'invite à remettre en question son propre rapport à l'environnement.

\section{Bibliographie}

Beltran-Vidal, Danièle. Chaos et renaissance dans l'œuvre d'Ernst Jünger. Berne : Lang, coll. « Contacts », 1995.

Descola, Philippe. «La forêt, lieu du sauvage ? Entretien avec Philippe Descola, par Anne de Malleray » Billebaude 5 (2014) : 4-11.

De Vriese, Hannes. «On the Meaning of Being Alone with Nature : Sylvain Tesson's Ecocritical Sincerity and Ecopoetic Sensuality in Dans les forêts de Sibérie ». French Ecocriticism. From the Early Modern Period to the Twenty-First Century. Dir. Daniel

A. Finch-Race et Stephanie Posthumus. Berne : Lang, 2017. 231-249.

De Feydeau, Bertrand. «La charpente de Notre-Dame de Paris n'est "pas reconstituable" : les arbres qui la composaient provenaient de la forêt primaire ». Franceinfo. 16 avril 2019. https://www.francetvinfo.fr/culture/patrimoine/incendie-de-notre-dame-deparis/la-charpente-de-notre-dame-de-paris-n-est-pas-reconstituable-les-arbres-qui-laconstituaient-provenaient-de-la-foret-primaire 3400799.html. Consulté le 16 avril 2019.

Harrison, Robert Pogue. Forests. The Shadow of Civilization. Chicago : U of Chicago P, 1992.

Hervier, Julien. Ernst Jünger. Dans les tempêtes du siècle. Paris : Fayard, 2014.

Jünger, Ernst. Le traité du rebelle, ou le recours aux forêts. Paris : Bourgois, 1995.

---. Eumeswil. Paris : La Table Ronde, 1978.

Le Bot, Jean-Michel. «Contribution à l'histoire d'un lieu commun : l'attribution à Chateaubriand de la phrase "les forêts précèdent les peuples, les déserts les suivent". 
Un exemple d'utilisation du logiciel Google Books Ngram Viewer ». Socio-logos 7 (2012). https://journals.openedition.org/socio-logos/2634. Consulté le 11 mai 2018.

Le Breton, David. Éloge de la marche. Paris : Métailié, 2000.

Lestel, Dominique. «Like the Fingers of the Hand. Thinking the Human in the Texture of Animality ». French Thinking About Animals. Dir. Louisa Mackenzie et Stephanie Posthumus. East Lansing, MI : Michigan State UP, 2015. 61-73.

Louis, Patrick. « Du rebelle à l'anarque ». Ernst Jünger. Dir. Philippe Barthelet. Lausanne :

L'Âge d'Homme, coll. « Les Dossiers H », 2000. 54-60.

Poussin, Alexandre. Marche avant. Guide à l'usage des aventuriers de grand chemin et des voyageurs immobiles. Paris : Laffont, coll. « Pocket », 2011.

Richard, Lionel. Le nazisme et la culture. Bruxelles : Complexe, 2006.

Schoentjes, Pierre. Ce qui a lieu. Essai d'écopoétique. Marseille : Wildproject, coll. «tête nue », 2015.

Tesson, Sylvain. Une très légère oscillation. Journal. 2014-2017. Paris : Équateurs, 2017.

---. Sur les chemins noirs. Paris : Gallimard, 2016.

---. Dans les forêts de Sibérie. Paris : Gallimard, coll. «Blanche », 2011.

---. Aphorismes sous la lune et autres pensées sauvages. Paris : Équateurs, 2008.

---. Éloge de l'énergie vagabonde. Paris : Équateurs, 2007.

---. Petit traité sur l'immensité du monde. Paris : Équateurs, 2005.

Verrier, Christian. Marcher, une expérience de soi dans le monde. Essai sur la marche écoformatrice. Paris : Harmattan, 2010.

Notes

1 «Les forêts précèdent les peuples, les déserts les suivent ». Bien que cette citation soit communément attribuée à Chateaubriand, il semble impossible de la localiser dans l'œuvre de l'écrivain, comme le signale Jean-Michel Le Bot dans son article "Contribution à l'histoire d'un lieu commun: l'attribution à Chateaubriand de la phrase "les forêts précèdent les peuples, les déserts les suivent" » (2012).

${ }^{2}$ Dès la première page de son journal Une très légère oscillation, Sylvain Tesson manifeste son enthousiasme pour la parution d'une biographie d'Ernst Jünger par Julien Hervier, qui retrace la vie du «mage » et « contemplateur»: «Enfin une année qui commence bien. [...] Le matin de la publication, je sors de la librairie en pressant le volume contre mon cœur » (13).

${ }^{3}$ On retrouve dans Le traité $d u$ rebelle d'Ernst Jünger les éléments feériques dont s'inspire Sylvain Tesson : «Voici le bois des contes, avec ses loups mangeurs d'hommes, ses sorcières et ses géants, mais où l'on trouve aussi le bon chasseur, les haies de roses de la Belle au Bois dormant, à l'ombre desquelles le temps suspend son vol » (75-76).

${ }^{4}$ La résistance de l'esprit est un concept qui a été récupéré de la notion politique d' "émigration intérieure », c'est-à-dire l'attitude de résistance passive d'intellectuel(le)s, d'écrivain(e)s ou d'artistes allemand(e)s sous le régime nazi. Lionel Richard la définit comme « l'attitude qui consista à rester en Allemagne tout en prenant ses distances à l'égard du national-socialisme et en refusant d'illustrer son idéologie par quelque œuvre que 
ce soit » (133). Après 1945, cette position a été dénoncée comme un prétexte invoqué par « ceux qui se sont réfugiés dans une littérature d'évasion » pour "protéger leur petite existence personnelle » (Richard 135). On pourrait arguer que si Sylvain Tesson se montre réticent (ou avoue son impuissance) à s'engager activement au profit de l'écologisme, son attitude se démarque toutefois par le nombre d'ouvrages publiés à ce sujet. Il est vrai que Tesson s'intéresse moins à Ernst Jünger l'écrivain politiquement controversé qu'à Ernst Jünger l'entomologiste, le botaniste et l'écologiste avant l'heure (la passion de Jünger pour l'univers des insectes et des fleurs trouve son écho dans l'ensemble de l'œuvre de Tesson).

5 À la suite de l'incendie du 15 avril 2019 qui a ravagé la cathédrale Notre-Dame de Paris et détruit la plus grande partie de la charpente, Bertrand de Feydeau, vice-président de la Fondation du patrimoine, a déclaré que cette «forêt » constituée de poutres en chêne datant du Moyen Âge n'était pas reconstituable. Il a expliqué que «nous n'avons plus sur notre territoire d'arbres d'une taille telle que ceux qui ont été coupés au treizième siècle et qui constituaient ce qu'on appelle la forêt primaire ». On estime que les arbres d'où provenaient les poutres de la première charpente de Notre-Dame pouvaient avoir entre trois cents et quatre cents ans lorsqu'ils ont été abattus. M. de Feydeau a expliqué en outre que si l'on voulait reconstruire la charpente de la cathédrale avec des arbres de la même dimension, on ne les trouverait pas sur le territoire français. Cette catastrophe est un rappel brutal du caractère irremplaçable de la nature primitive, et montre que la question de la préservation du patrimoine culturel de l'humanité peut s'associer aux enjeux environnementaux et à la conservation écologique des espèces et des habitats.

${ }^{6} \mathrm{Je}$ traduis de l'anglais : « an ecopoetic parenthesis ».

${ }^{7}$ Je traduis de l'anglais : « an existence that cannot be such except by feeding on the existences of other living beings and in allowing other beings to feed on it ».

${ }^{8}$ Je traduis de l'anglais : «infinite and personal debt ».

${ }^{9}$ Je traduis de l'anglais : «the obligations of reciprocity that jointly maintain humans and animals, but also plants, mushrooms, noncorporeal intelligences, etc. ».

${ }^{10}$ Tesson précise ailleurs : «Vivre, c'est préparer leur repas aux asticots » (Aphorismes, 126), ce qu'il considère comme un juste retour des choses compte tenu de l'élevage et de l'engraissement à grande échelle des animaux destinés à la consommation humaine. 